Reviews

\title{
Electrochemical DNA Detection Using Supramolecular Interactions
}

\author{
Shinobu Sato and Shigeori TAKENAKA ${ }^{\dagger}$ \\ Department of Applied Chemistry, Faculty of Engineering, Kyushu Institute of Technology, Kitakyushu, \\ Fukuoka 804-8550, Japan
}

\begin{abstract}
An electrochemical DNA detection format has been developed to allow simple and rapid assays with downsized instruments. Since naphthalene diimide derivatives are known to bind to double stranded DNA (dsDNA) with threading intercalation, they are stabilized by pseudo-catenane formation. For example, ferrocenylnaphthalene diimide (FND) can be efficiently concentrated on dsDNA, thus enabling its electrochemical detection. Since dsDNA is formed between single stranded target DNA and DNA probe, the target DNA can be detected electrochemically, using DNA probe-immobilized on the electrode. Further stabilization of naphthalene diimide derivatives as a complex with dsDNA on the electrode is expected to lead to more precise and selective detection of target DNA. This approach was realized by the formation of inclusion complexes of ferrocene with $\beta$-cyclodextrin $(\beta$-CD) for the FND-dsDNA complexes on the electrode. Combination of adamantylnaphthalene diimide (AND) and ferrocenyl- $\beta$-CD (Fc-CD) gave new supramolecular DNA detection assays coupled with DNA probe-immobilized electrodes. The electrochemical signal increased only upon formation of the Fc-CD/AND complex bound to dsDNA on the electrode. Naphthalene diimide carrying ferrocene and $\beta$-CD realized "signal on" type detection of dsDNA in homogenous solution. Naphthalene diimide carrying two dithiolene termini provided a new method to immobilize dsDNA on the gold electrode that may be suitable for the electrochemical detection of target DNA.
\end{abstract}

(Received April 3, 2012; Accepted May 9, 2012; Published July 10, 2012)

1 Introduction

2 Pseudo-catenane Formation between DNA Duplex and Threading Intercalator

3 Further Stabilization of the Complex of FND with Double Stranded DNA on the Electrode by $\beta$-Cyclodextrin

4 Supramolecular DNA Detection Using Adamanthylnaphthalene Diimide (AND) and Ferrocenyl- $\beta$-CD (Fc-CD)
643

646
5 "Signal on" Type Electrochemical DNA Detection by Naphthalene Diimide Carrying Ferrocene and $\beta$-CD

6 Naphthalene Diimide Carrying Dithiolane Moieties as DNA Stapler

7 Conclusion

648

8 Acknowledgements

9 References

\section{Introduction}

DNA detection is regarded as an important tool in the healthcare field. Some of its well-known healthcare applications include

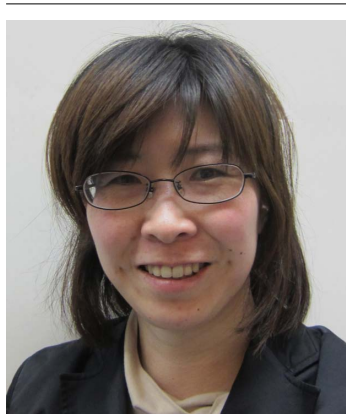

Shinobu SATo received her $\mathrm{PhD}$ in 2005 at Kyushu University. She worked at Kyushu Institute of Technology as Research Fellow of the Japan Society for the Promotion of Science (2005-2008) and as Assistant Professor of Research Center for Bio-microsensing Technology (2008 - 2010), and as Assistant Professor of Department of Applied Chemistry (2010 -). Her current research interest is the development of supramolecular DNA sensing technology.

\footnotetext{
To whom correspondence should be addressed.

E-mail: shige@che.kyutech.ac.jp

flu test, ${ }^{1}$ hepatitis screening, ${ }^{2}$ and drug susceptibility (studying side effects). ${ }^{3}$ Basically, DNA detection is mainly utilized through the use of fluorescence systems having fluorescent dyes specific for double stranded DNA (dsDNA). Such fluorescent dyes are also applied in Real-Time PCR, wherein the actual

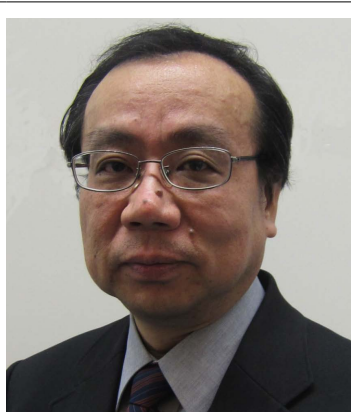

Shigeori TaKenaka received his $\mathrm{PhD}$ in 1988 at Kyushu University. He worked at Kyushu University as Research Associate (1987 - 1989) and as Associate Professor (1989 - 1991, 1996 - 2005). He also worked at Kyushu Institute of Technology (Kyutech) as Associate Professor (1991 1996) and as Professor (2005 -). He was a visiting scientist for Prof. W. David Wilson, Georgia State University (1994 1995). He is the director of the Research Center for Bio-microsensing Technology, Kyutech (2006-). His current research interest is the development of chemistry-based biosensing technology to achieve the practical use of biochips. 
(A)

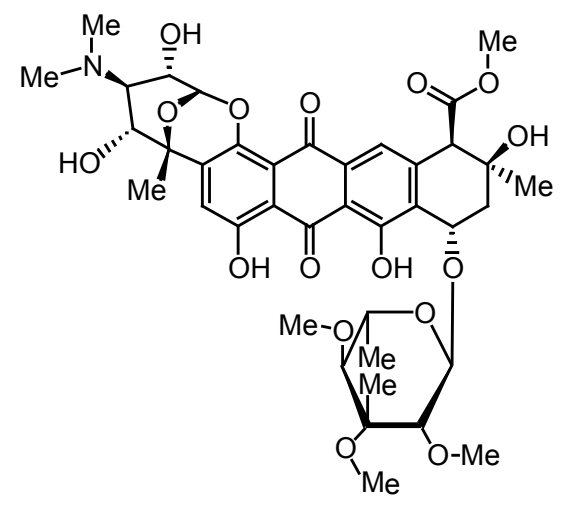

(B)

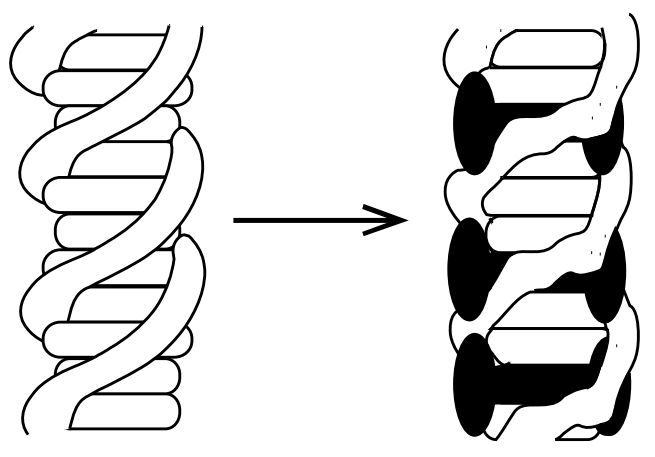

Fig. 1 (A) Chemical structure of nogalamycin and (B) its conceptual drawing as threading intercalator with dsDNA.

"real time" amounts of amplified dsDNA are estimated. Representative examples of fluorescent dyes widely used to detect dsDNA are: SYBRGreen, ${ }^{4}$ ethidium bromide, ${ }^{5}$ and PicoGreen. ${ }^{6}$ Many fluorescent dyes that have been developed through time have showcase high sensitivity and selectivity for dsDNA for the main purpose of DNA diagnosis in the early stage of the disease. Although each fluorescence system has its advantage, it falls short to some degree mainly for its high cost of operation which may not be often suitable for use especially in developing countries as a diagnostic tool. On the other hand, electrochemical DNA detection methods have demonstrated a great advantage in terms of higher sensitivity and selectivity for dsDNA, simple operation, low cost and easy downsizing, making them an attractive candidate for point of care testing (POCT) ${ }^{7,8}$ Electrochemical DNA detection system will thus have a widespread advantage once an electrochemical DNA ligand is developed that shows a higher preference for dsDNA. With this idea, we developed ferrocenylnaphthalene diimide (FND), which carries a high preference for dsDNA.9-11 Naphthalene diimide is a part of FND that binds with dsDNA and the ferrocene groups act as the electrochemical signaling labels. The complex formed between FND and dsDNA is stabilized through the catenane formation, resulting in high affinity of FND for dsDNA. Ferrocene has a reversible reduction and oxidation (redox) signal and the redox potential can be affected by changing the substituent function of the ferrocene unit. $^{10} \quad$ Furthermore, ferrocene is known to form the supramolecular complex in conjunction with $\beta$-cyclodextrin $(\beta-C D)$, which results in a change of electrochemical signal after complex formation. ${ }^{12}$
(A)

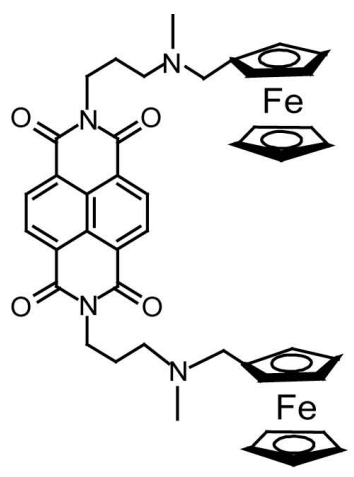

(B)

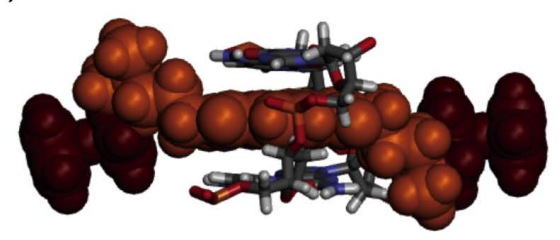

(C)

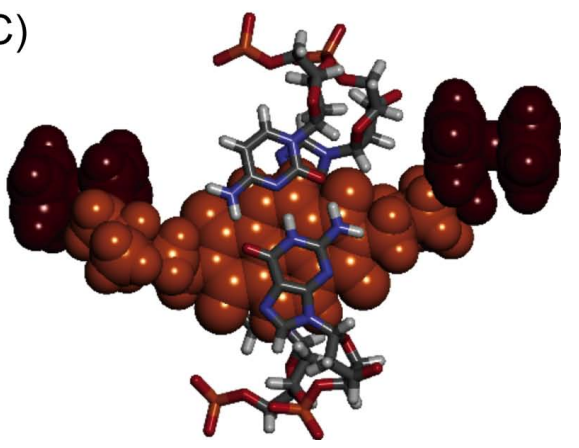

Fig. 2 (A) Chemical structure of ferrocenylnaphthalene diimide (FND), and (B) the side and (C) top view of the computer modeling of the threading intercalation complex of FND with dimeric dinucleotide. FND is shown as a CPK model.

We have constructed several supramolecular systems with the combination of dsDNA, naphthalene diimide, ferrocene and $\beta$-CD. ${ }^{11,13}$ The aim of this review paper is to compare and discuss our studies with the results reported by other research groups.

\section{Pseudo-catenane Formation between DNA Duplex and Threading Intercalator}

An intercalator is known as a DNA ligand, which slides or intercalates between adjacent base pairs of DNA duplexes. Nogalamycin or adriamycin as anticancer drugs, and ethidium bromide or methylene blue as fluorescence DNA detecting reagents are included in this category. ${ }^{5,14}$ Especially, nogalamycin is known to bind to DNA duplex with a unique intercalation mode, which is called "threading intercalation," since its two substituents are projecting out of both grooves of DNA duplex. ${ }^{15}$ One of the substituents of such threading intercalator needs to go through or thread into the adjacent base pairs to form a threading intercalation complex. This is a reason why such kind of interaction is called "threading intercalation." The complex thus obtained seems to possess the pseudo-catenane structure illustrated in Fig. 1. As can be expected for this 

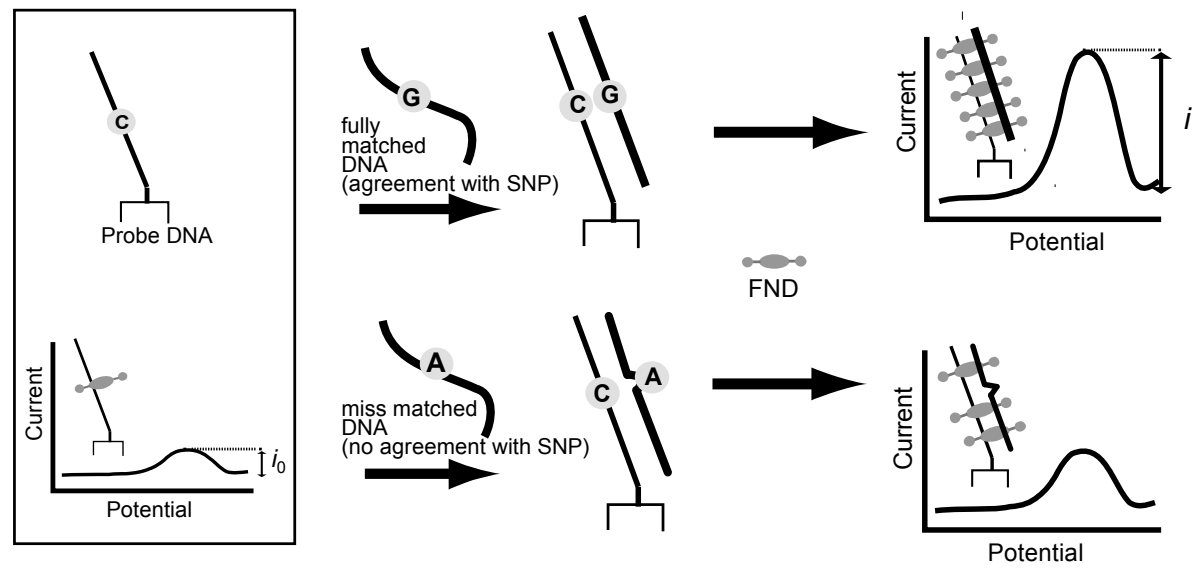

Fig. 3 Electrochemical DNA detection by the FND-based hybridization assay. The concentration of FND intercalated to DNA on the electrode was decreased with the reduced number of base pairs of DNA. Current increase percentage $(\%),\left(i / i_{0}-1\right) \times 100$.

structure, the intercalation complex should exhibit a very slow dissociation rate due to the presence of two anchoring substituents. Ferrocenylnaphthalene diimide (FND) (Fig. 2) as a threading intercalator was shown to exhibit 135-times slower dissociation rate than that for ethidium bromide. ${ }^{15}$ The stabilization by threading intercalator should be observed only for dsDNA, but not for ssDNA. This feature of threading intercalation is expected to be useful to discriminate between dsDNA and ssDNA targets.

We have developed an electrochemical gene detection technique using FND as an electrochemical threading intercalator. $^{9-11}$ FND binds preferentially to dsDNA with threading intercalation mode. When this intercalator was applied to electrochemical hybridization assay using DNA probe-immobilized electrode, target DNA formed the hybrid with DNA probe on the electrode and it could be detected as dsDNA on the electrode through the electrochemical signal of FND concentrated on the electrode (Fig. 3).

We have applied this system to develop FND-based hybridization assay. Use of this system permitted the mismatched DNA discrimination for detection of single nucleotide polymorphism (SNPs) in several assays: in lipoprotein lipase gene, which is linked with arteriosclerosis; ${ }^{16}$ in the gene connected with the side effect of anticancer drug, irinotecan; ${ }^{17}$ in tumor-suppressor gene, $\mathrm{p} 53 ;{ }^{18}$ and in aberrant methylation of $\mathrm{p} 16^{19}$ or $\mathrm{CDH} 4$ gene $^{20}$ that is expected to serve as a cancer marker.

We have also analyzed the effect of DNA density and its length on the hybridization efficiency in FND-based hybridization assay (Fig. 3). Oligonucleotide sample carrying the same length as probe DNA (24-meric DNA) was allowed to hybridize with DNA probe-immobilized electrode (Fig. 3A). ${ }^{20}$ Hybridization efficiency decreased from 100 to $60 \%$ with an increase in DNA probe density on the electrode from $1 \times 10^{12}$ to $2 \times 10^{12}$ molecules $/ \mathrm{cm}^{2}$ and reached plateau at a probe density exceeding $6 \times 10^{12}$ molecules $/ \mathrm{cm}^{2}$. Under $6 \times 10^{12}$ molecules $/ \mathrm{cm}^{2}$ of DNA probe density on the electrode, the average distance between two DNA probes was estimated as $2 \mathrm{~nm}$. Therefore, steric hindrance during hybridization process should cause the decreasing of hybridization efficiency.

On the other hand, hybridization efficiency of 121-meric PCR products was less than $20 \%$ under probe density range of $1 \times 10^{12}-6 \times 10^{12}$ molecules $/ \mathrm{cm}^{2}$. This low hybridization efficiency derived also from steric repulsions in the hybridization process between the hybridized region located in the middle of 121 mer and the DNA probe on the electrode. However, an increase in current around $60-100 \%$ was observed for both oligonucleotide sample and PCR products under FND-based hybridization assay. Since many FNDs were concentrated to target DNA hybridized with DNA probe on the electrode, the electrochemical signal was observed effectively even at low hybridization efficiency. Under aberrant methylation of $\mathrm{CDH} 4$ gene, hybridization behavior of PCR products was studied after bisulfite treatment. ${ }^{20}$ FND-based hybridization assay of this sample gave a detection limit of $0.5 \mathrm{ng} / \mu \mathrm{L}$ for PCR product. The results of this assay using electrodes carrying different DNA probes were in agreement with sequencing results. ${ }^{20}$

\section{Further Stabilization of the Complex of FND with Double Stranded DNA on the Electrode by $\beta$-Cyclodextrin}

Under FND-based hybridization assay as shown in Fig. 3, electrochemical response of the DNA probe-immobilized electrode was measured in the electrolyte containing FND before and after hybridization with target DNA. The electrochemical response was observed also before hybridization; this signal came from FND concentrated on DNA probe due to electrostatic or stacking interactions between FND and phosphate anion or nucleic base, respectively. To decrease background signal of FND, one should reduce concentration of FND in the assay, which requires improved stability of the dsDNA/FND complex. According to this strategy, we tried to further stabilize the complex of FND with dsDNA using $\beta$-cyclodextrin (CD). The $\alpha-, \beta$-, and $\gamma$-CD are known to form inclusion complexes with ferrocene molecule ${ }^{12}$ and $\beta$-CD forms 1:1 complex with the ferrocene molecule possessing the binding constant of $2200 \mathrm{M}^{-1} .{ }^{12}$ Thus, we attempted to stabilize the complex of FND with DNA duplex by adding $\beta$-CD, as shown in Fig. $4 .{ }^{11}$ We tested the binding affinity of FND with dsDNA in the absence and the presence of $\beta$-CD; the binding constant in the presence of $\beta$-CD was $6.0 \times 10^{5} \mathrm{M}^{-1}$, which was twice that observed in the absence of $\mathrm{CD}$. The obtained kinetic parameters of the binding process showed that this increase in binding constant derived from the diminished dissociation rate 
(A)

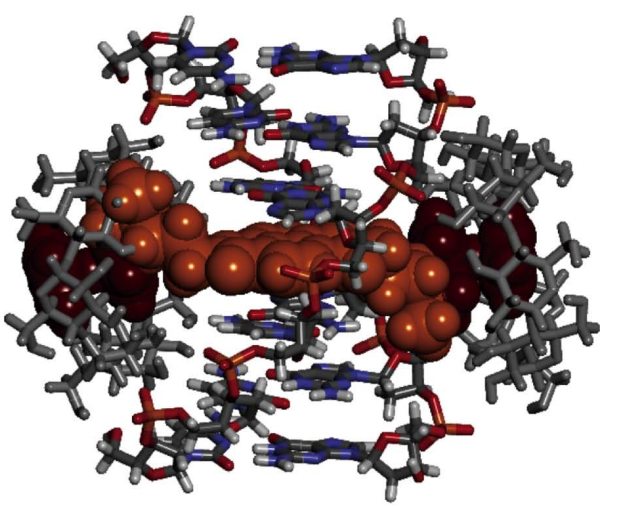

(B)

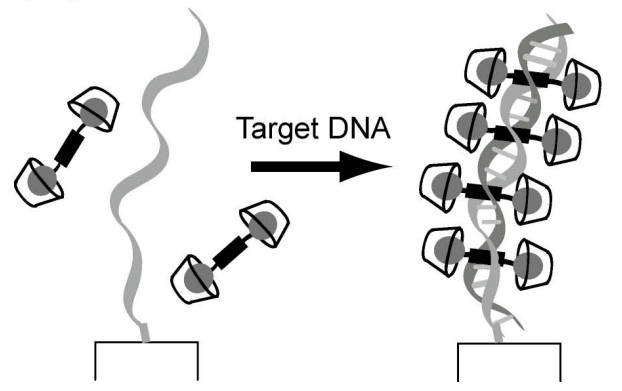

Fig. 4 (A) Computer modeling of an inclusion complex of FND-dsDNA with $\beta$-CD and (B) strategy of DNA detection by using FND and $\beta$-CD. FND is shown as a CPK model.

constant of FND caused by the stabilization effect of $\beta$-CD on the FND/dsDNA complex.

The potential of the redox peak of FND observed in cyclic voltammograms of dsDNA-immobilized electrode at $0.1 \mathrm{M}$ AcOK-AcOH and $0.1 \mathrm{M} \mathrm{KCl}$ containing $50 \mu \mathrm{M}$ FND underwent a positive shift upon addition of $\beta-\mathrm{CD}$, that was accompanied with a decrease in peak current. The difference between oxidation and reduction potentials, $\Delta E_{\mathrm{p}}$, of $21 \mathrm{mV}$ indicated that the concentration of FND on the electrode proceeded through binding to dsDNA, but not through adsorption on the gold surface. ${ }^{21}$ This result gives a proof of ternary complex formation of FND with double stranded DNA and $\beta-\mathrm{CD}$, where $\beta$-CD include ferrocene moieties of FND intercalated with double stranded DNA.

Finally, we tested FND-based hybridization assay in the presence of $\beta$-CD using 24 mer DNA probe-immobilized electrode for 24-meric sample DNA with complementary or non-complementary sequence under the electrolyte containing $800 \mu \mathrm{M} \beta$-CD, $50 \mu \mathrm{M}$ FND, $0.1 \mathrm{M}$ AcOK-AcOH, and $0.1 \mathrm{M}$ $\mathrm{KCl}$ (Fig. 5). Peak current responses of 0.5 and $1.2 \mu \mathrm{A}$ were observed before and after hybridization with complementary sample DNA; the obtained current increasing percentage was $140 \%$. The same experiment was carried out in the absence of $\beta$-CD; the peak current responses of 2.2 and $3.9 \mu \mathrm{A}$ were obtained before and after hybridization with complementary sample DNA and the obtained current increasing percentage was $80 \%$. Finally, we succeeded in increasing the discrimination ability of target DNA using $\beta$-CD or supramolecular complex formation. However, the current response of FND bound to double stranded DNA was decreased by the inclusion of ferrocene with $\beta$-CD.

As a next step, we constructed a system with ferrocene jumping out of $\beta$-CD cavity after formation of supramolecular

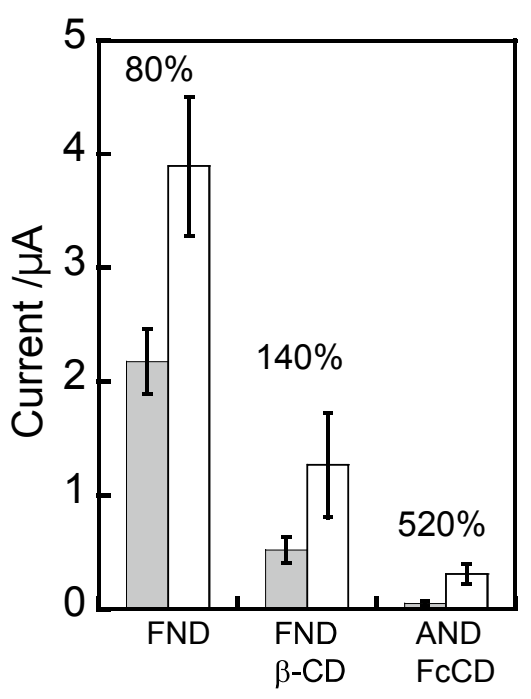

Fig. 5 Peak currents before (gray histograms) and after hybridization (white histograms) with complementary DNA in the cases of FND, $\mathrm{FND}+\beta-\mathrm{CD}$, and AND + Fc-CD systems.

complex induced by hybridization of target DNA and DNA probe on the electrode.

\section{Supramolecular DNA Detection Using Adamanthylnaphthalene Diimide (AND) and Ferrocenyl- $\beta$-CD (Fc-CD)}

Adamantane is known to be good guest for $\beta$-CD with $10^{5} \mathrm{M}^{-1}$ of binding affinity. ${ }^{22} \quad$ Ferrocene-modified $\beta$-cyclodextrin $(\mathrm{Fc}-\mathrm{CD})$ is also known to form intramolecular inclusion complex of ferrocene moiety with cyclodextrin cavity in the aqueous solution, resulting in its diminished redox current. ${ }^{22}$ However, when adamantane was added to a solution containing Fc-CD, the ferrocene was replaced with adamantane and ferrocene jumped out from $\beta$-CD, resulting in increased redox current. Thus, we synthesized adamanthylnaphthalene diimide (AND) and applied it to increase electrochemical signal in DNA detection coupled with Fc-CD. ${ }^{13}$ Design strategy and working principle of this system are shown in Fig. 6. When DNA duplex was formed on the electrode between target DNA and DNA probe, the supramolecular complex was formed by AND, Fc-CD, and DNA duplex. This is expected to generate the current increase as shown in Fig. 6.

AND shows the binding constant of $2.0 \times 10^{5} \mathrm{M}^{-1}$ for dsDNA, which is twice that of FND in the absence of $\beta$-CD. Circular dichroism spectrum of Fc-CD showed the positive induced $\mathrm{CD}$ signal around $450 \mathrm{~nm}$; this ICD band disappeared upon addition of AND, suggesting that the intramolecular inclusion of ferrocene with $\beta$-CD was kicked off by AND. Target DNA carrying 24 mer DNA was allowed to hybridize with 24 mer DNA probe on the electrode and the electrochemical gene detection was achieved with this supramolecular system containing $50 \mu \mathrm{M}$ AND, $100 \mu \mathrm{M}$ Fc-CD, $0.1 \mathrm{M}$ AcOK-AcOH, and $0.1 \mathrm{M} \mathrm{KCl}$. The peak current values of 50 and $310 \mathrm{nA}$ were observed before and after hybridization, respectively, resulting in the current increase percentage of $520 \%$ (Fig. 5). 
(A)

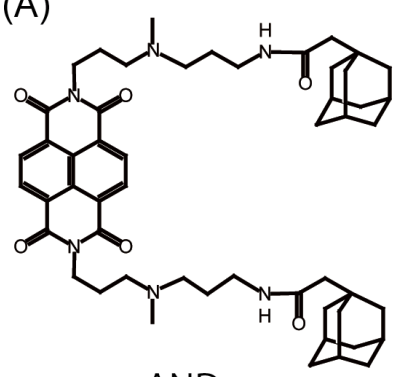

AND

(B)

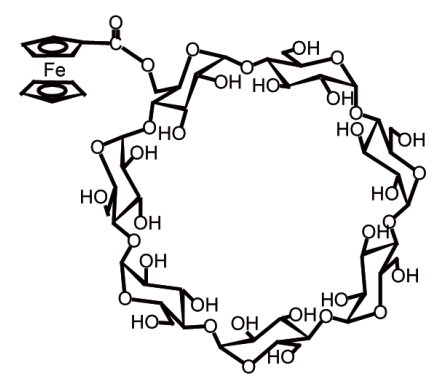

Fc-CD

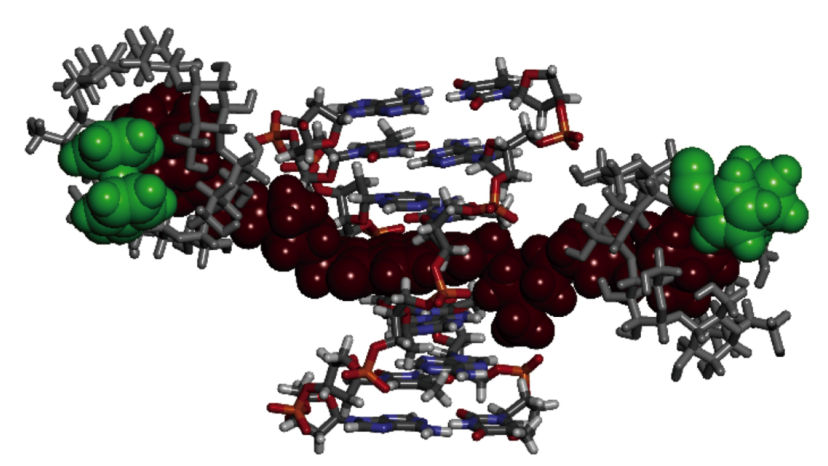

(C)

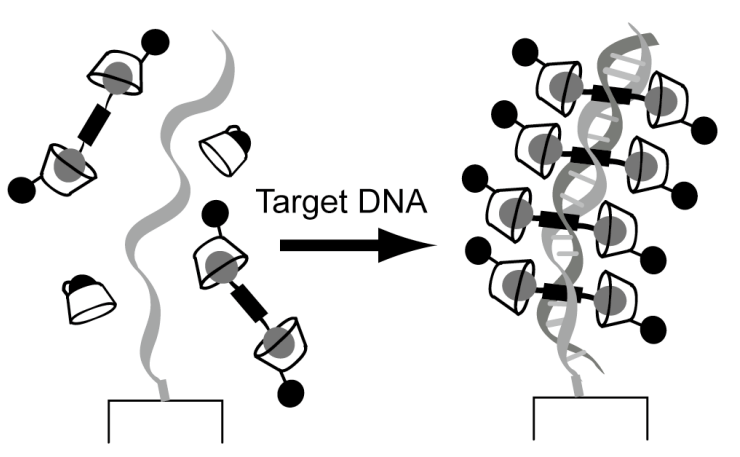

Fig. 6 (A) Chemical structure of AND and $\mathrm{Fc}-\mathrm{CD}$, (B) computer modeling of the complex between AND, Fc-CD, and dsDNA, and (C) strategy of DNA detection by using AND and Fc-CD. AND and Fc parts are shown as CPK models.

\section{5 "Signal on" Type Electrochemical DNA Detection by Naphthalene Diimide Carrying Ferrocene and $\beta$-CD}

We tried to develop electrochemical detection of dsDNA in homogenous solutions using electrochemically active intercalators. Recently, electrochemical real time dsDNA detection has been reported using electrochemically active intercalator derivatives. ${ }^{23-25}$ The dsDNA was detected by the decrease of the diffusion coefficient of redox intercalator upon binding to dsDNA, resulting in the current decrease. This system is regarded as a homogenous electrochemical dsDNA assay, but it is "signal off"-type detection. We tried to construct the "signal on" type detection system using ferrocene and $\beta$-CD. The principle is shown in Fig. 7.26 Synthesized naphthalene diimide carrying ferrocene and $\beta$-CD (FNC) showed the intramolecular inclusion structure in homogenous solution; the current of ferrocene was diminished and shifted towards positive potential comparing with free ferrocene. However, ferrocene jumped out from $\beta$-CD upon binding to dsDNA and the current

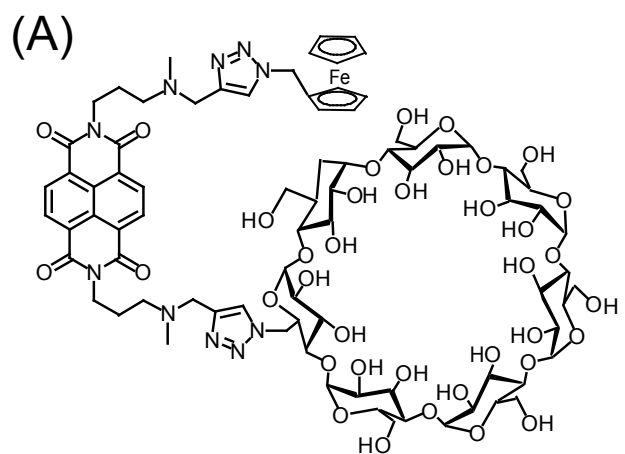

(B)

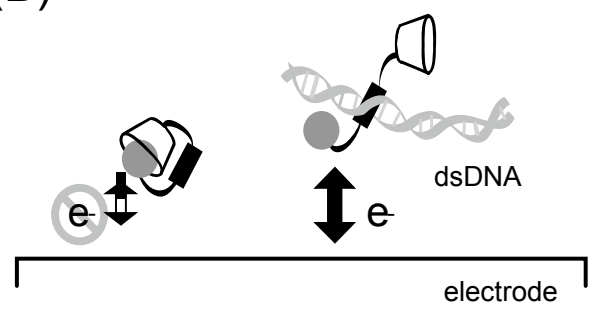

(C)

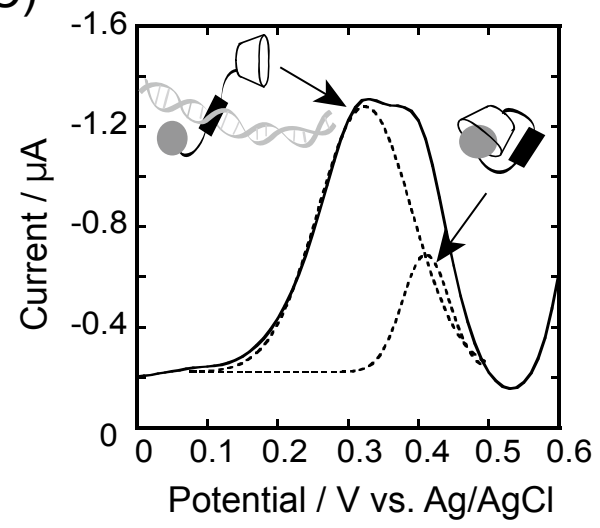

Fig. 7 (A) Chemical structure of FNC, (B) the concept of dsDNA detection using FNC in homogenous solution, and (C) differential pulse voltammogram of FNC in the presence of dsDNA. Two overlapped peaks were observed, which were curve-decovoluted into that for DNA complex and that for FNC alone.

signal was expected to increase with an increase in the amount of dsDNA with a negative potential shift. Scatchard analysis using the spectrum change of FNC upon addition of dsDNA gave binding constant of $8.9 \times 10^{4} \mathrm{M}^{-1}$. CD spectra of FNC upon addition of dsDNA suggested the threading intercalation as a binding mode. Cyclic voltammograms of FNC in the absence and the presence of dsDNA showed reduction peaks at 420 and $320 \mathrm{mV}$; the peak at $420 \mathrm{mV}$ decreased with DNA addition, whereas that at $320 \mathrm{mV}$ increased. Experiments including the competition assay between ferrocene and adamantane showed that the peak at $420 \mathrm{mV}$ should be assigned to the ferrocene included by $\beta-\mathrm{CD}$, while that at $320 \mathrm{mV}$ represented the ferrocene alone. The intensity of the current peak at $320 \mathrm{mV}$ depended on the concentration of dsDNA and "signal on" type electrochemical detection of dsDNA was achieved by the monitoring the peak current at $320 \mathrm{mV}$. This system was applied to detect PCR products; it showed the detection limit of $26 \mathrm{nM}$ of target PCR product. Furthermore, AFM images suggested that the intramolecular inclusion 
(A)<smiles>O=C(CCCCC1CCSS1)NCCCN1CCN(CCCN2CCN(CCCN3C(=O)c4ccc5c6c(ccc(c46)C3=O)C(=O)N(CCCN3CCN(CCCCC4CCSS4)CC3)C5=O)CC2)CC1</smiles>

(B)

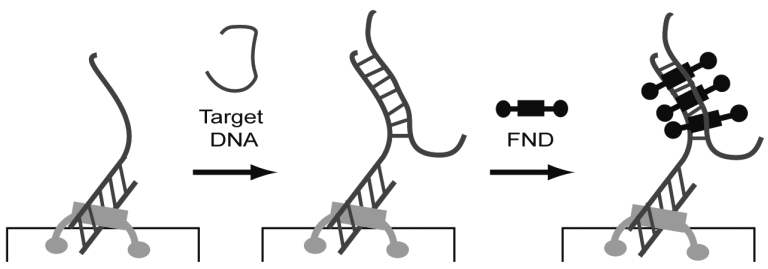

Fig. 8 (A) Chemical structure of NDI-ss, (B) concept of the FNDbased hybridization assay with the use of probe DNA-immobilized by NDI-ss.

complex of FNC was dissociated when FNC intercalated into dsDNA.

\section{Naphthalene Diimide Carrying Dithiolane Moieties as DNA Stapler}

Synthesized naphthalene diimide having two dithiolane moieties at its substituted termini, NDI-ss, was reported to bind to dsDNA by threading intercalation. ${ }^{27}$ When this resulting complex was applied to a gold surface, this complex was immobilized on the gold surface through a dithiolane-gold linkage. The conceptual scheme of this immobilized complex is shown in Fig. 8. The NDI-ss molecule seems to act as a molecular stapler to immobilize a dsDNA. DNAs from several sources were immobilized on the gold surface using NDI-ss as long as dsDNA region was present. FND-based hybridization assay was carried out using DNA probe immobilized on the gold electrode, which was prepared by NDI-ss and the oligonucleotide having 24-meric single stranded probe region and 21-meric double stranded region as shown in Fig. 9. Since NDI-ss bind to double stranded regions of the oligonucleotide, single stranded regions can be used as probes to hybridize with target DNA. Hybridization efficiency for target DNA was $92 \%$ under the immobilization density of $8 \times 10^{12}$ molecules $/ \mathrm{cm}^{2}$. Hybridization efficiency was only $10-50 \%$ using the oligonucleotide carrying thiol moiety at its terminal, which is known as common technique, although the same immobilization density of DNA probe was used. This result showed that hybridization efficiency in the case of DNA probe using NDI-ss was higher than that using thiol-modified DNA probe. It is expected that former DNA probe laid on the gold electrode surface, whereas the latter DNA probe stood up on the electrode, so the target DNA could more easily access in the case of the former under hybridization. FND-based hybridization assay of 124-meric target DNA was carried out for the above DNA probe-immobilized electrode prepared by DNA probe carrying partial double stranded region with NDI-ss and thiol-modified DNA probe under similar
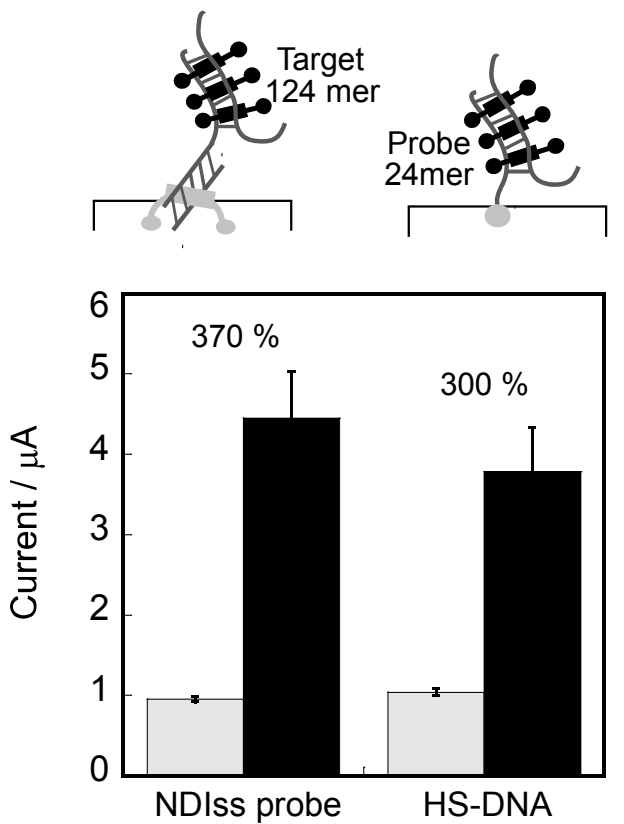

Fig. 9 Current response of electrode modified with 24-meric DNA probe immobilized with NDI-ss or directly attached through thiol moiety before (gray histograms) and after hybridization (black histograms) with 124-meric complementary DNA strand.

density of immobilized DNA probe. Current increases were 370 and $300 \%$ in FND-based hybridization using DNA probe electrode prepared by the former and the latter procedure, respectively. This result reflected the hybridization efficiency in both cases.

\section{Conclusion}

Electrochemical DNA detection using supramolecular interactions was described in this review. Supramolecular complex formation between ferrocene and $\beta$-CD is interesting because of the electrochemical behavior changes after complexation. DNA detection using supramolecular systems with other groups have been also reported. Ihara et al. prepared two DNA probes carrying ferrocene and $\beta-\mathrm{CD} .^{28}$ These probes formed the complex between ferrocene and $\beta$-CD after hybridization only with target DNA. Aoki et al. prepared DNA probe carrying ferrocene and $\beta$-CD at both termini. ${ }^{29}$ This approach is a "signal on" type system, where intramolecular complex between ferrocene and $\beta$-CD was destroyed by the complex formation after hybridization with target DNA, which is similar to our detection system using FNC. These electrochemical assays were achieved in homogenous solution. Recent research moves from DNA immobilized systems to homogenous ones, which is good for real time DNA detection system although these are "signal off" type approaches. ${ }^{16-18}$ Thus, the "signal on" type DNA detection using electrochemical technique is expected to open new fields in biosensing technology.

\section{Acknowledgements}

This work was supported in part by a Grant-in-Aid for Scientific Research from the New Energy and Industrial Technology 
Development Organization (NEDO) and Grants-in-Aid for Scientific Research from the Ministry of Education, Science and Culture of Japan.

\section{References}

1. T. J. Falla, D. W. M. Crook, L. N. Brophy, D. Maskell, J. S. Kroll, and E. R. Moxon, J. Clin. Microbiol., 1994, 32, 2382.

2. H. Okamoto, H. Tokita, M. Sakamoto, M. Horikita, M. Kojima, H. Iizuka, and S. Mishiro, J. Gen. Virol., 1993, 74, 2385.

3. F. Innocenti, L. Iyer, and M. J. Ratain, Am. Soc. Drug Metabol. Dispos., 2001, 29, 596.

4. M. W. Pfaff, Nucleic Acids Res., 2001, 29, e45.

5. M. G. Murray and E. F. Thompson, Nucleic Acid Res., 1980, 8, 4321.

6. V. L. Singer, L. J. Jones, S. T. Yue, and R. P. Haugland, Anal. Biochem., 1997, 249, 228.

7. X. Luo and I.-M. Hsing, Analyst, 2009, 134, 1941.

8. N. J. Ronkainen, H. B. Halsall, and W. R. Heineman, Chem. Soc. Rev., 2010, 39, 1747.

9. S. Takenaka, K. Yamashita, M. Takagi, Y. Uto, and H. Kondo, Anal. Chem., 2000, 72, 1334.

10. S. Sato and S. Takenaka, J. Organomet. Chem., 2008, 693, 1177.

11. S. Sato, T. Nojima, M. Waki, and S. Takenaka, Molecules, 2005, 10, 693.

12. T. Matsue, D. H. Evans, T. Osa, and N. Kobayashi, J. Am. Chem. Soc., 1985, 107, 3411.

13. S. Sato, T. Nojima, and S. Takenaka, J. Organomet. Chem., 2004, 689, 4722.

14. E. M. Tuite and J. M. Kelly, J. Photochem. Photobiol., B, 1993, 21, 103.
15. S. Takenaka and M. Takagi, Bull. Chem. Soc. Jpn., 1999, 72, 327.

16. K. Yamashita, A. Takagi, M. Takagi. H. Kondo, Y. Ikeda, and S. Takenaka, Bioconjugate Chem., 2002, 13, 1193.

17. S. Sato, Y. Maeda, T. Nojima, H. Kondo, and S. Takenaka, Nucleic Acids Res., 2003, 3(Suppl.), 169.

18. H. Miyahara, K. Yamashita, M. Kanai, K. Uchida, M. Takagi, H. Kondo, and S. Takenaka, Talanta, 2002, 56, 829.

19. S. Sato, H. Kondo, K. Hokazono, T. Irie, T. Ueki, M. Waki, T. Nojima, and S. Takenaka, Anal. Chim. Acta, 2006, 578, 82

20. S. Sato, M. Tsueda, Y. Kanezaki, and S. Takenaka, Anal. Chim. Acta, 2012, 715, 42.

21. A. J. Bard and L. R. Faulkener, "Electrochemical Methods: Fundamentals and Applications", 2nd ed., 2001, John Wiley and Sons, New York, Chichester, Brisbane, Toronto.

22. A. Ueno, O. Chen, I. Suzuki, and T. Osa, Anal. Chem., 1982, 64, 1650.

23. T. Defever, M. Druft, D. Evrard, D. Marchal, and B. Limoges, Anal. Chem., 2011, 83, 1915.

24. B. Y. Won, S. Shin, S. Beak, Y. L. Jung, T. Li, S. C. Shin, D.-Y. Cho, S. B. Leecd, and H. G. Park, Analyst, 2011, 136, 1573.

25. K. Yamamura, M. Saito, K. Kondo, M. M. Hossain, R. Kotetsu, T. Saeki, T. Nagatani, K. Ikuta, and E. Tamiya, Analyst, 2011, 136, 2064.

26. S. Watanabe, S. Sato, K. Ohtsuka, and S. Takenaka, Anal. Chem., 2011, 83, 7290.

27. S. Sato, A. Hirano, and S. Takenaka, Anal. Chim. Acta, 2010, 65, 91.

28. T. Ihara, T. Wasano, R. Nakatake, P. Arslan, A. Futamura, and A. Jyo, Chem. Commun., 2011, 47, 12388.

29. H. Aoki, A. Kitajima, and H. Tao, Supramol. Chem., 2010, 22,455 . 